\title{
La construction de l'éthique de l'entreprise ou éthique des affaires Première Partie. Les formes à partir de l'adoption de l'éthique*
}

\author{
Ricardo Cuevas Moreno **
}

\section{Résume}

Dans cet article nous montrons la façon de l'entreprise ou l'éthique des affaires se construit à partir de la contribution de la science de l'éthique. Cela est à l'origine des formes dont nous nommons: l'entreprise du respect et de la bonne volonté, la voix du prophète et l'élan d'amour, Habermas dans la gestion, la solution de la contrainte morale et le transfert des valeurs de l'éthique à l'entreprise. L'analyse des formes illumine le contenu idelogique de l'éthique de l'entreprise et les contradictions insurmontables dans sa construction. Pour ce faire, nous mobilisons la méthode de l'économie politique, de Marx (1965, 1857: 254-263) en notre exposé sur la construction de l'éthique de l'entreprise. De cette façon nous continuons le parcours établi par la méthode de l'économie politique de Marx (1857) en nôtre l'exposé de la constitution de l'éthique de l'entreprise à la française.

Mots clés : éthique de l'entreprise, éthique des affaires, éthique, idéologie, management participatif, projet personnel, éthique de la discussion, éthique professionnel, travail immatériel éthique de la conviction et éthique de la responsabilité.

\footnotetext{
* Ce travail est composé de deux parties. La deuxième partie sera présentée dans le prochain numéro de cette revue.

**Investigador de la Facultad de Comercio y Administración de Tampico de la Universidad Autónoma de Tamaulipas. Correo electrónico: rcuevasfr@yahoo.fr
} 


\section{Introduction}

\section{L'interprétation française de l'éthique de l'entreprise ou l'éthique des affaire}

T es articles suivants sont consacrés aux formes que l'éthique de l'entreprise revêt. L Notre analyse se limite à l'interprétation française de l'éthique de l'entreprise parce qu'elle est un fait particulier depuis son adoption et son développement en France. Au fil du temps, l'éthique de l'entreprise a été assimilée et interprétée en accord avec la culture propre de ce pays. Dans ce sens, il nous semble important de souligner les points suivants: (1) Une conception propre des rapports entre l'Etat, les patrons et les travailleurs. (2) Un contexte social, économique et politique particulier. (3) Une tradition philosophique qui essaie de soutenir tout le discours de l'éthique de l'entreprise. Toutefois, nous pensons que notre analyse peut servir à comprendre certains aspects de l'éthique de l'entreprise en général, notamment son rôle comme idéologie du capital.

L'éthique de l'entreprise est nommée de différentes manières : L'éthique dans la gestion de l'entreprise, l'éthique des affaires, l'éthique dans l'entreprise. Nous avons décidé de la nommer simplement l'éthique de l'entreprise ou l'éthique des affaires. La raison en est double: d'une part, les écrivains de l'éthique de l'entreprise se réfèrent de façon indistincte à un même objet d'analyse ; d'autre part, cette notion englobe tous les rapports de l'entreprise (internes et externes). Ces rapports sont pour nous économiques, moraux, mais aussi politiques, c'est-à-dire d'autorité et de pouvoir. Etant donné que l'entreprise fait partie de l'organisme social, la notion d'éthique de l'entreprise englobe aussi les rapports internes et externes de l'entreprise capitaliste. Enfin, nous partons du fait que l'entreprise capitaliste est la forme dominante de l'entreprise dans le monde actuel.

L'éthique de l'entreprise repose sur une série de concepts et de raisonnements qui lui rendent son contenu et sa validité. Par exemple, ceux que nous nommons les écrivains de l'éthique de l'entreprise supposent la société capitaliste libérale et ses valeurs politiques et morales comme point de départ. Ils sont le cadre de tout discours. Nous pensons que l'intégration des valeurs morales faite pour l'éthique de l'entreprise ou l'éthique des affaires est une porte d'entrée pour expliquer son caractère idéologique.

En conséquence l'analyse desformes donne la preuve que l'éthique de l'entreprise ou l'éthique des affaires est un idéologie du capital dans le sens de Marx et Engels 
(1982, 1846). La forme est la façon dont le contenu d'un processus où phénomène se structure. L'idéologie est la forme (représentation) que acquièrent les rapports sociaux de propriété, de production et de pouvoir dans une société divisée en clases. L'idéologie prend l'apparence de science et se pressente commun à tous. En cachant les rapports qui le rendent son fondement, l'idéologie est une fausse conscience avec l'apparence de vérité. De cette façon l'idéologie perpétue les rapports d'exploitation et de domaine d'une classe sur les autres. Dans la pratique la idéologie impose les idées, les valeurs de la classe social qui domine les mondes économique et intellectuelle moyennant la Philosophie, le Droite la Politique, la Éthique, la Religion.

Le problème est d'expliquer comment une idéologie, celle de la classe dominante, réussit à s'imposer au reste de l'ensemble social. Á notre époque, la connaissance scientifique semble être la forme la plus efficace que l'idéologie assume. Toutefois, en tant qu'idéologie, elle peut prendre une autre forme, par exemple, de discours religieux.

Dans cet article nous verrons la façon dont l'éthique de l'entreprise emprunte les connaissances de l'éthique pour justifier son existence, son discours et ses recommandations. Compte tenu que l'éthique, l'économie et la gestion sont les sources de la justification dont nous parlons, nous tâchons de mettre d'abord, en évidence l'apport de la science de l'éthique. Ensuite, l'apport de la science de l'économie à l'éthique de l'entreprise, nous le réservons pour le article suivant puisque cela débouche sur le dogme qui soutient toute l'éthique de l'entreprise, celui qui postule que "les valeurs morales sont la clé de réussite de l'entreprise».

\section{L'adoption de l'Éthique par l'éthique de l'entreprise}

Les écrivains de l'éthique de l'entreprise justifient le besoin de l'orientation éthique pour la gestion de l'entreprise de plusieurs manières :

- Les hommes travaillant dans l'entreprise, notamment l'entrepreneur et le gestionnaire doivent prendre des décisions. Cela implique la responsabilité et partant, la morale.

- Les changements dans le monde actuel obligent de plus en plus à ce que l'entreprise intervienne dans les affaires de la société et cela implique les questions morales : L'entreprise citoyenne est la figure emblématique decette position. 
- L'entreprise actuelle, forme décentralisée et en réseau, libère des espaces d'autonomie pour les individus où la communication devient de plus en plus importante pour la gestion : l'entreprise du troisième type ou l'entreprise intelligente sont les figures représentatives.

Les deux livres pionniers en France ont inauguré une démarche commune à la littérature de l'éthique de l'entreprise. Après avoir établi le besoin de l'éthique pour les affaires, M. M. Gélinier (1991) et Farcy (1969) font intervenir la pensée d'un philosophe à partir duquel les recommandations pour une éthique de l'entreprise sont valables. Nous analyserons cette articulation.

\section{a. L'éthique de l'entreprise et les théories éthiques}

Le premier exemple de cette articulation tire de la théorie éthique de Kant $(1785,1994)$ sa base. Les auteurs de Vers une morale des affaires (Farcy, 1969) formulent une question qui semble typique pour l'éthique de l'entreprise : en partant de l'homme libre, capable de réaliser des choix, comment peut-il devenir conscient et responsable vis-à-vis de la prise de décisions et de ses conséquences ? En bref, comment l'homme peut-il avoir une attitude morale dans les affaires? Commet l'homme peut concilier le respect vers les autre dans la prise de décisions efficaces pour accomplir les objectifs de l'entreprise?

A la suite de Farcy, Moussé (1989) Etchegoyen (1991) et Bowie (1999) abordent cette problématique. Ils proposent des solutions adoptant la théorie éthique de Kant $(1785,1994)$. Ces réponses supposent l'individu raisonnable, autonome et muni de droits universels.

D'après l'auteur, la nature des obligations de chacun se détermine par les droits des autres. M. Farcy pense, comme les écrivains de l'éthique de l'entreprise le feront par la suite, queles droits de l'homme tendent à l'universalité et énoncent de façon explicite la reconnaissance de la dignité et le respect de la personne ; ils sont le cadre juridique, politique pour l'économie de marché. Il pense qu'il s'agit de trouver dans le cadre de la société libérale, la meilleure façon pour le fonctionnement de l'économie de marché. Les droits et les devoirs de l'homme appliqués à la morale commerciale permettent l'accomplissement de la liberté, de l'équité, de la justice et de la sécurité des individus dans les contrats et les transactions (Farcy, 1969 : 41-49). 
De façon pratique, M. Farcy recommande une pédagogie de la décision dans laquelle l'homme apprend à se juger soi-même et à se fixer des priorités. Enfin, pensons-nous, la pédagogie de la décision rendra possible de concilier la subjectivité de l'individu et son devoir moral envers les autres. De cette façon, l'antinomie entre l'intérêt particulier et l'intérêt général sera résolue. Autrement dit, l'attitude éthique rendra compatibles l'efficacité économique et la morale.

C'est ainsi, pensons-nous, que dans l'éthique de l'entreprise inspirée de Kant, les hommes auront la bonne volonté pour respecter les engagements dans l'accomplissement des rapports de la production et de la circulation sociale de l'entreprise. L'éthique de Kant appliquée à l'entreprise prônerait pour un respect de la personne, dans lequel les différents partenaires devraient se considérer toujours comme une fin jamais comme un simple moyen (Kant, 1785,1994: 108). Autrement dit, cette application ne résoudrait le problème de l'application pratique de l'impératif catégorique qu'en oubliant la divergence des intérêts dans l'entreprise.

L'application de la morale de Kant à l'entreprise met en évidence, pensons nous, le besoin de recourir à l'accomplissement du devoir et à l'éthique née des Lumières pour expliquer les rapports moraux dans l'entreprise.

Le deuxième exemple Gélinier (1991) et (Even-Granboulan, 1988) mobilisent certes concepts et idées de Durkheim (1937) et Bergson (1932) sur l'éthique, la morale et son apprentissage et le comportement moral dans l'entreprise. Nous pensons que la application de ces théories éthique pour la gestion de l'entreprise mènerait vers les réflexions suivantes.

Nous sommes êtres moraux dans la mesure que nous sommes sociaux. L'accomplissement du devoir renvoie aux notions d'autorité et de discipline. La morale est un ensemble de devoirs, de habitudes et de commandements ; elle est un système d'interdits, lequel a par but mettre des limites aux passions et borner la sensibilité en accord aux ordres de la raison. La moral est essentiellement discipline (Durkheim 1937: 27,30, 36-37). L'apprentissage de la morale se donne au travers de l'éducation et la convivialité dans les groupes sociaux. La pression social et l'élan d'amour -qui son les manifestation complémentaires de la vie- sont les deux notions basiques qui expliquent le besoin de l'obéissance et la morale pour préserver le groupe et la société (Bergson 1932: 52-53; 98-99). 
Sous cette perspective l'entreprise serait une institution et un groupe. L'attachement de l'homme à ce groupe particulier serait l'essence du devoir et de l'acte moral. L'autorité et la discipline expliqueraient l'établissement des normes, la soumission et l'accomplissement du devoir quotidien. L'obligation morale, comme nous l'avons vu chez Durkheim, serait le résultat de l'autonomie de la volonté. En bref, la vie morale pour l'entreprise synthétiserait le bonheur, le bien et le devoir. L'entreprise serait à la fois le groupe et l'éducatrice d'une morale rationnelle et laïque.

Mais ni Gélinier (1991), ni (Even-Granboulan, 1988) dérivent les implications que ces théories éthiques ont pour la gestion de l'entreprise. Ils usent seulement quelques concepts et idées de Bergson et Durkheim pour justifier certains conseilles de ce que les affaires devraient être. (Gélinier, 1991: 9,13, 50-51, 56, 69, 75, 78, 155-156, 161-165, 175-178, 183) (Even-Granboulan, 1988: 95, 99, 130-136, 191, 222, 248, 262, 269, 277). Cela est une carácter de l'éthique des affaires.

Habermas dans la gestion est le troisième exemple. Pour l'entreprise du nouveau millénaire ou l'entreprise du troisième type, en utilisant l'expression d'Archier et Sérieyx (1984) l'information et la communication sont devenues les activités cruciales pour la vie des entreprises. L'importance gagnée par le travail immatériel se traduit en une mutation des entreprises et de leur gestion. Alors, la gestion des hommes et des moyens de la production doit s'effectuer par un nouveau type de gestion, le management participatif.

La vie nouvelle des entreprises demande de l'action autonome des individus et l'identification de leur projet de vie avec l'entreprise. Les conflits que cette étape des entreprises génère doivent être résolus en prenant en compte une société libérale démocratique avancée. L'éthique de Habermas $(1983,1992)$ semble être adéquate pour l'entreprise actuelle. Sauf, que les écrivains de l'entreprise oublient certaines questions essentielles de l'éthique de Habermas.

L'éthique de la communication se trouve dans un programme de recherche plus ample. Il s'agit de l'acceptation du mode de vie capitaliste et de la domination sur les masses de ce mode de production. Dans ce sens, la légitimation du capitalisme et de l'entreprise et l'acceptation de la part de la masse est une affaire cruciale. Les écrivains de l'éthique de l'entreprise oublient que la communication et l'information jouent un rôle fondamental pour la légitimation et la domination de l'entreprise capitaliste moderne. Mais cela est en plus une 
parcelle que Habermas même n'exploite pas (Floris, 1996). Par exemple, (Etchegoyen, 1991 : 184, 185,186, 187-188) offre une application limitée de la théorie de la communication d'Habermas.

D'ailleurs, comment peut-on être sûr de ne pas exclure certains acteurs ou groupes de la discussion? (Leroux y Marciano, 1999). De notre part, nous pensons à une autre objection. Car l'éthique de la discussion suppose des hommes libres, égaux, raisonnables et responsables dont le dialogue est basé sur les principes « $\mathrm{U}$ » et « D » (Habermas, 1986, 1983: 12-13). Ces hommes en discutant feront émerger les normes valides et acceptées par tous (Habermas, 1992, 1991: 17-32). Cela signifierait la possibilité de parvenir à une démocratisation de la vie de l'entreprise inconnue jusqu'à nos jours.

Le quatrième exemple use la notion de vertu comme fondement des affaires. Selon Solomon (2000) les affaires ne peuvent pas être limités au fait de produire l'argent. En revanche, la pratique des vertus éthiques, qui pour lui sontles valeurs éthiques, se trouve dans le cœur des affaires. Cela implique que les êtres humains sont qui donnent vie aux affaires plus que les profits ou l'argent. Les êtres humains donnent le succès aux entreprises et aux affaires. Solomon, autant qu'Aristote, part de l'individu dans sa communauté dont critère de succès es le bonheur. La vie bonne, c'est la vie heureuse qui fleurit en agissant bien, c'est-à-dire une vie guidée par les vertueux.

L'essence de la démarche d'Aristote vers l'éthique des affaires est que nous devons nous écarter de la pensée centrée en les utilités et les concevoir comme une partie fondamental de la vie bonne. (Solomon, 2000, 1999: xxiii)

Selon Solomon l'individualisme, la liberté individuelle, la responsabilité collective, le respect et la tolérance son les valeur - plus que la liberté de libre entreprise sans rendre comptes à personne - sont à l'origen du succès des entreprises des EtatsUnis. Pour cet auteur il faut humaniser les affaires. Bannir de nos conceptions et de notre langage les métaphores de la jungle, le jeu, la guerre, la machine; le mythe de l'entrepreneur, la concurrence et le capitalisme de cow-boy, l'altruisme abstrait et les utilités.

La démarche de Solomon basée en Aristote, postule qu'être vertueux implique agir en bénéfice personnel et en bénéfice des autres. L'idée sous-jacente, nous pensons, est que l'homme existe seulement dans la communauté. Cela, est certainement 
commun aux sociétés Grecque de l'Antiquité et la société capitaliste. Mais ce fait servit à Solomon pour établir que la vie dans l'entreprise existe avant tour par et en la communauté. Cela est l'argument ontologique qui mène à la morale. Le deuxième argument est de nature politique et supplémente le premier. Selon Solomon l'entreprise est a "citoyenne" car, il est part et vit en société. En conséquence, l'entreprise visé comme une citoyenne et comme communauté permet la compréhension des activité des affaires et de ses aspects étique» (Solomon, 2000, 1999: 34, 46).

D'après Solomon la vertu qui synthétise à toutes, est l'intégrité. Laquelle est identifiée par lui avec le caractère, c'est-à-dire la faculté d'être honnête, de respecter et penser en les autres dans chacune de nos actions. Finalement, les versus sont telles, seulement en rapport aux autres, c'est là qu'elles acquièrent la qualité de l'utilité. En effet, inspiré en Hume $(1751,1991)$ et son Enquête sur les principes de la morale, Solomon pense que les vertus de l'entreprise et la société sont nécessairement utiles (Hume, 1991, 1751: 128). Dans ce sens la responsabilité sociale de l'entreprise est inhérente à la vie de l'entreprise autant que son utilité par le bien de la société.

\section{b. La solution de la contrainte morale}

Le cinquième exemple de l'articulation entre l'éthique et l'éthique des affaires est remarquable par ses répercussions dans la construction de cette idéologie du capital. C'est pour cela que nous en ferons une étude spéciale. D'abord, nous analysons la exposée de Weber sur l'éthique de la conviction et l'éthique de la responsabilité. Puis, nous analyserons l'interprétation et l'application dans l'éthique des affaires de cette célèbre maxime.

Dans ses conférences de 1919, Weber essaie de solutionner la portée éthique des vocations du savant et du politique. Ces deux activités ont en commun le fait qu'elles ne reposent pas sur un vide social. Elles impliquent les aspects d'ordre social, politique, économique, historique et éthique que les hommes consacrés à la science et à la politique doivent prendre en compte dans l'exercice de leur vocation. Il est évident que le jeu de ces facteurs dépasse le simple exercice de la vocation. Dans les deux conférences, la vocation du savant et celle du politique arrivent à un problème éthique commun : celui de la portée de la science et celui de la politique pour le destin de la société et de l'humanité. 
La célèbre maxime formulée par $\mathrm{M}$. Weber, l'éthique de la conviction et l'éthique de la responsabilité sont reprises maintes fois pour expliquer le comportement moral pratique des agents. Dans la présentation du livre Le savant et le politique, R.Aron (Weber, 2001, 1919) soutiennent l'idée que cette formule reflète dans une grande mesure la condition humaine. Certes, cela semble être l'intention de Weber.

Le contexte où cette formule a été énoncée, pensons-nous, permet de mieux comprendre sa signification. La vocation $d u$ savant et la vocation du politique sont comprises à partir d'un système capitaliste développé. Là, les institutions comportent les qualités de l'administration rationnelle, c'est-à-dire des « entreprises capitalistes » dont l'objectif premier est le gain. En effet, Weber suppose le capitalisme avancé : la spécialisation des activités et la tendance à convertir en entreprises capitalistes toutes les activités de la vie sociale.

Le politique auquel pense Weber, est l'homme qui se consacre à temps complet à la politique et qui vit pour la politique. Un des résultats remarquables du processus d'établissement de l'Etat moderne est l'homme politique professionnel. Il existe l'homme politique en tant que fonctionnaire et l'homme politique en tant que chef, leader, entrepreneur de l'affaire politique. Celui-ci est différent du fonctionnaire à temps complet. Le fonctionnaire gère les ressources de l'administration; sa réussite est l'administration efficace et responsable de ces biens.

Autant que le savant, le politique (le chef politique, le professionnel, l'homme d'Etat) consacre sa vie au service d'une cause : pour le premier c'est la science, pour le deuxième, le pouvoir. Le politique met ses doigts dans les rayons de la roue de l'histoire. Et cela mène Weber à établir la question suivante : quelles sont les qualités nécessaires au politique pour être à la hauteur de cette intervention? Autant que le savant, le politique doit être muni d'un ensemble de qualités indispensables pour atteindre son but. Weber mentionne la passion sincère, la responsabilité personnelle (dérivée de l'engagement à la cause) et le coup d'oeil —faculté psychologique, faculté de distance des faits, des hommes et des choses.

Selon M. Weber, la légitimité de la domination comporte des fondements. Elles sont aussi les trois raisons internes qui justifient la domination : l'autorité qui naît de la tradition, l'autorité qui provient du charisme et l'autorité qui provient de la légalité ou de la raison, tout simplement. 
Pour les rapports entre la politique et l'éthique oul'éthos de la politique, la question décisive est la suivante : la politique est une activité des efforts dans le but d'obtenir le pouvoir. C'est l'exercice de la violence légitime qui se trouve au cœur de la politique, de l'Etat moderne et de l'ethos de la politique pour la vie humaine. La cause que sert l'homme politique est la prise ou le partage du pouvoir pour lui, pour un parti ou pour un groupe.

Etant donné que selon Weber, le moyen décisif en politique est la violence, alors, qu'est-ce qui peut justifier l'usage de la force de la part des hommes de la société sur d'autres? Si nous considérons les moyens et les buts, qu'est-ce qui peut justifier que dans le but d'atteindre des fins bonnes ou valables l'homme politique ou l'Etat utilisent la violence légitime ou des moyens moralement dangereux ? Plus encore, qu'est-ce qui peut justifier que les conséquences de ces actes de force soient fâcheuses ou moralement dangereuses? D'après Weber, aucune éthique ne peut résoudre ces questions. En plus, le résultat final de l'activité politique correspond rarement aux intentions premières de l'acteur. Ce sont les conséquences non voulues des actes des hommes qui aboutissent aux résultats non prévus qui nous accompagnent, au moins, depuis la naissance de monde moderne.

Face à cette impossibilité de l'éthique pour répondre aux paradoxes de la vie humaine, Weber établit le fait que toute activité orientée selon l'éthique peut être subordonnée aux deux maximes: l'éthique de la conviction et l'éthique de la responsabilité. Elles sont des principes de caractère général irréductiblement opposés. Toutefois, cela ne veut pas dire que dans l'éthique de la conviction se trouve absente l'éthique de la responsabilité et vice versa.

Il est vrai que M. Weber ne définit pas exactement ces deux éthiques. Ce sont les exemples qu'il fournit qui permettent d'apercevoir leur signification. Il s'ensuit que la caractéristique fondamentale de l'éthique de la conviction c'est que l'homme, guidé par elle, ne prend pas en compte les conséquences prévisibles de ses actions. Si les actes tirent des conséquences fâcheuses, le partisan de l'éthique de la conviction les attribuera à la sottise des hommes ou encore à Dieu. Du point de vue logique, l'éthique de la conviction repousse toute action qui fait appel aux moyens moralement dangereux. Weber décrit le partisan de l'éthique de la conviction comme quelqu'un qui ne supporte pas l'irrationalité du monde. Il est un "rationaliste cosmo-éthique». Mais dans la réalité, la volte-face brusque caractérise le partisan de l'éthique de la conviction : il peut prôner la non-violence et l'amour et tout à coup basculer dans la violence justifiée pour qu'on atteigne la 
paix complète. En revanche, selon Weber, le partisan de l'éthique de la responsabilité prend en compte les conséquences prévisibles de ses actes. Dans le cas de l'homme politique, cela est très net. Il s'expose aux conséquences de ses actions. L'usage de la violence légitime est le point crucial de ses actes et de ses conséquences. Le cas du militant religieux ou politique qui combat par conviction met aussi en évidence cette thèse.

D'après Weber, dans l'activité politique, la lutte idéologique guidée seulement par l'éthique de la conviction peut aboutir à des dommages considérables parce qu'elle est démunie de la responsabilité des conséquences. Malgré cette distinction, on ne peut conseiller à personne d'agir guidé par l'éthique de la conviction ou l'éthique de la responsabilité et dans quel moment utiliser l'une ou l'autre.

Or, le politique auquel songe Weber, pensons-nous, est la synthèse et le juste équilibre de l'éthique de la conviction et de l'éthique de la responsabilité. Il est vrai qu'au départ de son exposé, Weber présente ces deux éthiques comme irréconciliables. Mais, quelques passages après, il résulte que chez le politique, elles peuvent se compléter :

On le voit maintenant : l'éthique de la conviction et l'éthique de la responsabilité ne sont pas contradictoires, mais elles se complètent l'une l'autre et constituent ensemble l'homme authentique, c'est-à-dire un homme qui peut prétendre à la vocation politique. (Weber, 2001, 1919 : 219)

\section{c. Le gestionnaire : saint ou diable}

L'adoption que les écrivains de l'entreprise faite de la formule éthique de Weber se présente comme une explication et un outil dans la prise de décisions. Les choix du gestionnaire peuvent être analysés à partir de l'éthique de la conviction et de l'éthique de la responsabilité. Dans ce sens, l'antinomie éthique de Weber est contenue par un même individu. Elle représente un moment de tension entre les convictions personnelles et les responsabilités que l'entreprise lui demande. M. Orsoni dans son article L'éthique en gestion (Leroux et Marciano: 1999) établit qu'appliquée à l'entreprise, l'éthique de la responsabilité conduirait parfois le gestionnaire à effectuer des actions moralement condamnables dans le but d'atteindre les objectifs de l'entreprise. L'éthique de la responsabilité appliquée aux gestionnaires est essentiellement fondée sur la recherche de l'efficacité. 
En revanche, l'éthique de la conviction appliquée au gestionnaire vise toujours un idéal sans prendre en compte les conséquences des actes pour l'entreprise.

Une autre interprétation que M. Orsoni mentionne (Leroux et Marciano, 1999: 197-205) de la dichotomie éthique de Weber, est celle de l'homme d'action et le philosophe, le saint ou le prophète. L'éthique de la responsabilité est liée au premier et l'éthique de la conviction au deuxième. D'après M. Moussé (1993) ce clivage existe dans la réalité. L'homme d'action et le philosophe ont une vision limitée de la réalité. Le premier cherche des résultats en accord à l'efficacité ; sa vision est pragmatique et utilitariste. Le philosophe comporte une activité plus théorique. Donc, ce qui manque à l'homme d'action, ce sont les outils théoriques qui lui permettent de comprendre les causes de son comportement dans la prise de décisions. Les hommes d'affaires ont une formation et un mode de vie qui leur empêche la réflexion sur la morale ou l'éthique de leur activité ; leur formation quantitative absorbe l'attention de ceux-ci sur les mesures planes et les projets (Moussé, 1993: 11-12, 40, 56).

Compte tenu qu'il existe une "multiplicité d'éthiques et une multiplicité d'appréciations » du bien et du mal, pour Moussé (1993) cette totalité est insaisissable. Alors, selon lui, la question importante est de savoir comment s'articulent les différents niveaux moraux des individus dans l'entreprise même, autrement dit, les niveaux des responsabilités professionnelles, des responsables de l'entreprise et leurs convictions personnelles. Enfin, l'articulation de l'éthique dans l'entreprise (Moussé, 1993 : 40, 137, 139-140).

M. Moussé pense que la coopération conflictuelle de la vie de l'entreprise mène indéfectiblement à la célèbre dichotomie de Weber entre l'éthique de la conviction et l'éthique de la responsabilité (Moussé, 1993: 141-144). Cet auteur propose l'exercice de la liberté responsable comme la solution à ce conflit. Solution insatisfaisante, pensons nous, parce qu'elle recoure à la conscience individuelle et au libre arbitre dont la bonne volonté de l'individu joue le premier rôle. Autrement dit, en arrière plan, la dichotomie éthique établie par Weber a pour soutien l'éthique de Kant $(1994,1785)$ malgré que Moussé refuse l'éthique de l'entreprise inspirée à partir de cette éthique.

En effet, la dichotomie de l'éthique de la conviction et de l'éthique de la responsabilité est une maxime qui prétend avoir une validité générale et orienter ainsi, la vie morale des hommes. Elle représente l'opposition abyssale des deux attitudes de 
la vie morale. La caractéristique - la seule que Weber distingue nettement - est la prise en compte ou non des conséquences des actes de la part de l'individu. Car, on suppose autant que Weber, des hommes libres et rationnels, c'est-à-dire des hommes qui peuvent choisir entre une éthique de la conviction et une éthique de la responsabilité. D'ailleurs personne ne peut les obliger à pratiquer l'une ou l'autre.

Supposons que l'éthique de la conviction et l'éthique de la responsabilité se trouvent l'une et l'autre à deux extrêmes. Dans la pratique les hommes pourraient osciller entre ces deux extrêmes en s'éloignant de l'une ou de l'autre. Il est vrai que la différence et les vertus entre ces deux éthiques s'apprécient clairement aux extrêmes. L'interprétation de M. Aron (Weber, 2001, 1919) considère en effet, que cette antinomie reflète le cas extrême de la condition humaine. Or, nous pensons que le contenu de la maxime de Weber comporte un élément psychologique sans lequel elle ne serait pas valable.

Certes, dans la vie immédiate, l'antinomie de Weber entre ces deux éthiques apparaît indubitable. A la surface de la société, l'attitude des hommes varie d'une éthique de la conviction à une éthique de la responsabilité et d'une éthique de la responsabilité à une éthique de la conviction. Il est vrai aussi qu'elles peuvent être interpénétrées, mélangées dans les cas concrets. Ainsi si nous traçons une ligne entre ces deux éthiques en les plaçant l'une et l'autre à l'extrémité de chaque bout, nous verrons que lors qu'un homme s'éloigne de l'une, il tend vers l'autre; tout dépend du cas et de l'individu. Alors, l'homme politique auquel pense Weber, devrait avoir tendance à rester au centre de cette ligne dans le cas idéal.

Mais il semble pertinent de se demander quel est l'élément qui relie ces deux extrêmes. Nous pensons que les deux extrêmes (celle de l'éthique de la conviction et celle de l'éthique de la responsabilité) sont reliées par un facteur commun : le devoir. L'homme de l'éthique de la conviction et l'homme de l'éthique de la responsabilité agit en accord au devoir. La différence est que l'un envisage les conséquences des décisions et l'autre pas. M. Weber ne s'occupe pas de connaître l'origine de leur conscience morale, ni l'origine des valeurs autour desquelles les deux éthiques tournent. En revanche, il est bien conscient que les conflits naissent de la structure même de la société.

En effet, nous savons en accord à l'histoire de l'éthique, que le devoir et son accomplissement appartiennent à la réalisation du monde des valeurs morales. De plus, ces valeurs existent et sont réalisées par les hommes d'une société historique 
définie. Dans une société divisée en classes, les valeurs dominantes sont les valeurs de la classe moralement dominante. L'accomplissement du devoir est une condition de la reproduction de la vie sociale. Alors, l'éthique de la conviction et l'éthique de la responsabilité essaient de montrer l'attitude, la manière dont les hommes accomplissent leur devoir. C'est-à-dire l'attitude que les agents prennent en réalisant leur conscience morale.

Car dans la vie pratique, les hommes se voient dans la contrainte de choisir entre l'accomplissement de leurs convictions ou celui de leurs responsabilités. Weber veut leur fournir un principe pratique de repérage dans cette perpétuelle contrainte. La maxime de Weber est de caractère psychologique et subjective. Il a confiance, pensons-nous, dans le fait que les hommes auront le bon jugement pour discerner l'éthique de la conviction de l'éthique de la responsabilité. De plus, les hommes ont la possibilité de réussir le juste équilibre entre l'une et l'autre. Cela est d'autant plus important chez l'homme politique qui exerce la violence légitime sur les autres hommes ; il affecte les ressources, gère le destin des nations et des Etats. Sauf, que l'éthique de la responsabilité et l'éthique de la conviction laissent un grand horizon de possibilités pour relativiser toutes les positions.

Cela peut s'expliquer à partir de deux choses : D'abord parce que selon Weber, l'éthique ne peut pas justifier l'usage de moyens moralement condamnables pour atteindre des fins bonnes. Alors, l'éthique de la conviction et l'éthique de la responsabilité seraient les uniques repères possibles des choix des individus. En conséquence, la science de l'éthique ne pourrait jamais expliquer le sens du comportement moral, pas plus qu'elle ne pourrait en énoncer des lois. Ce qui resterait c'est le choix des agents sans aucune loi. L'interprétation de M. Wunenburger (1993) dans ses Questions d'éthique considère qu'avec la maxime morale, Weber essaie de démontrer l'impossibilité qu'ont les hommes à agir à partir des valeurs morales établies d'avance.

Mais, nous savons que les valeurs morales et sociales sont des données à partir desquelles les hommes accomplissent leur vie morale et leur devoir, même s'ils ne connaissent pas leur origine. Les valeurs sont des figures familières et à la fois méconnues par l'homme de la masse. Ils ne le savent pas mais reproduisent ces valeurs; et ils le font entre ce qui dicte leur conscience morale particulière et ce qui dicte leur conscience morale sociale. La maxime de Weber, pensons-nous, considère l'attitude des individus dans l'activité morale. L'antinomie, éthique de la conviction et éthique de la responsabilité — comme l'appelle M. Aron—serait la 
forme pratique que prendrait la conscience morale. Les pôles de cette contradiction et sa connexion sont analysés à partir de la rationalité. D'après ceci, les deux éthiques seraient les deux formes de la conscience morale rationnelle.

C'est pour cela que pour nous, (en supposant un moment que l'antinomie éthique de Weber soit valable) l'identification de l'homme d'action à l'éthique de la responsabilité, et du savant ou du philosophe à l'éthique de la conviction est une opposition fictive que nous ne trouvons pas chez Weber. Dans la vie pratique, l'homme d'action et le savant vivent, tendent, soit vers l'éthique de la conviction, soit vers l'éthique de la responsabilité. Il est différent de dire que l'homme d'action doit préférablement agir guidé par l'éthique de la responsabilité, comme dans le cas du gestionnaire. Et que le cas idéal serait un juste équilibre entre les deux éthiques comme Weber le préconise pour le politique. Dans ce sens, ce qui est abyssal ce sont les deux éthiques, et non plus les activités des individus liées de façon fictive à l'antinomie éthique de Weber.

De plus, on ne peut pas associer, tout simplement, l'éthique de la conviction à la morale du cœur et l'éthique de la responsabilité à la morale de la raison; comme s'il s'agissait de deux éthiques dont l'origine serait distincte : l'une l'instinct, l'autre la raison. Avec ceci, on prend le risque inutile d'établir une opposition de nature différente que celle que Weber établit. Il en va de même si on prend la métaphore de Dieu et du Diable; le bien et le mal, pour les associer, soit l'éthique de la conviction, soit l'éthique de la responsabilité. C'est une métaphore que Weber utilise pour illustrer l'opposition entre ces deux éthiques et la relativité de ce qui peut être considéré comme bon ou mauvais. Au-delà de la science il n'y a que des choix. Les hommes sont confrontés à des choix où la science ne peut pas leur porter secours. Ainsi, dans le monde règne les décisions individuelles des agents qui choisissent leurs propres dieux et leurs démons.

Dans ce sens, la dichotomie de Weber appliquée au gestionnaire dirait que celui-ci n'est ni dieu ni le diable, ni bon ni mauvais. Selon M. Orsoni (1989) le gestionnaire accomplirait rarement la morale de la responsabilité jusqu'aux dernières conséquences, si cela impliquait l'utilisation des moyens moralement fâcheux. En revanche, une pure application de la morale de la conviction dans l'entreprise est impraticable. La solution proposée par M. Orsoni, pensons-nous, est plus fidèle à la pensée de Weber. En effet, dans la pratique le gestionnaire et les affaires se déroulent entre les deux extrêmes des deux morales: 
Au fond, la morale des affaires évolue entre ces deux extrêmes, car de toute évidence, il n'y a pas d'éthique de la responsabilité qui ne reste imprégnée par une conviction exprimée ou latente. (Orsoni, 1989, núm. 74, juin : 114)

Compte tenu des observations que nous avons faites sur la dichotomie éthique de Weber (l'absence de l'origine des valeurs morales, son caractère subjectif, et le devoir comme la substance et le lien entre les deux éthiques) et l'interprétation de M. Orsoni (1989) pour l'éthique de l'entreprise nous obtenons le schéma suivant :

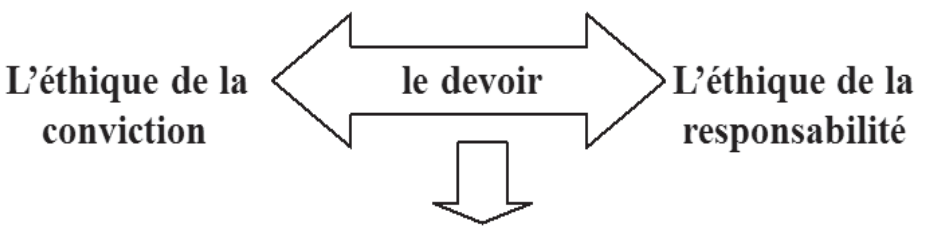

Le gestionnaire

(l'homme)

Il représente un idéal, un juste équilibre entre l'accomplissement des convictions et des responsabilités. Dans la pratique, le gestionnaire autant que le politique penchera pour un côté ou pour l'autre. L'éthique de l'entreprise de M. Orsoni comporte une perspective pratique et un contenu humain. Donc, la morale du gestionnaire serait le fruit d'un apprentissage, d'une recherche avec endurance du bien pour les hommes qui travaillent dans l'entreprise :

L'exercice de la morale reste un effort de discernement du bien et du mal .... Le problème de la morale de l'entreprise est celui de l'homme et le problème du gestionnaire consiste à chercher, non pas le Bien absolu, mais à chaque fois, pas à pas, un bien pour l'homme.

(Orsoni, 1989, núm. 74, juin : 109-116)

En somme, d'après M. Orsoni (Leroux y Marciano, 1999: 197-205) le gestionnaire est partagé entre la conduite instrumentale qui vise les objectifs et les résultats de la firme et la conduite du cœur qui vise ses valeurs et ne prend pas en compte les contraintes ni le déterminisme des événements. Si on évite la fausse identification de l'éthique de la conviction et de l'éthique de la responsabilité avec une morale du cour et une morale de la raison absentes chez Weber, cette distinction de M. Orsoni peut éviter la confusion de mots.

Or, une autre façon d'expliquer que le gestionnaire n'est ni dieu ni le diable ; et que le problème éthique dans l'entreprise va plus au-delà $d u$ bien ou $d u$ mal, c'est 
l'enseignement de Marx (1965, 1867: 550) qui ne culpabilise pas les actions des individus dans le monde économique où ceux-ci ne sont que des créatures. Les actions du gestionnaire sont déterminées par l'organisme économique et les buts de l'entreprise dans des rapports caractérisés par l'autorité, le pouvoir et la quête du profit. Dans ce sens, la conscience morale de l'individu et ses convictions, sont soumises aux lois de l'organisme social non pas comme phénomène isolé mais comme tendance nécessaire du mode de vie capitaliste (Marx, 1965, 1867: 49).

Finalement, nous considérons qu'il existe un point crucial dans la célèbre antinomie de Weber : L'impossibilité éthique de justifier dans la quête de fins bonnes, des moyens et des conséquences moralement dangereuses. Ce problème est avant tout un problème pratique : les fins déterminent les moyens mais les moyens déterminent aussi les résultats des fins. L'histoire de la vie sociale même est la preuve la plus claire : les fins et les moyens ne sont jamais moralement neutres. Cela est plus évident quand il s'agit de légitimer la violence de certains hommes sur d'autres; les licenciements massifs ou les mesures de politique économique comme dans le cas des pays du tiers monde, qui adoptent la politique économique suggérée par le FMI. Là, les responsabilités et les convictions les plus éclairées ou les plus abjectes sont passées par le crible du mouvement social et de l'histoire.

M. Aron (Weber, 2001, 1919) établit le défit qui infirmerait les thèses de Weber. Mais Aron formule mal les choses. La science sociale ne comporte jamais la vérité totale, ne prédit pas parfaitement l'avenir ni ne promet la résolution des conflits et des valeurs des communautés de l'avenir. La science sociale explique les raisons de l'existence des faits et leurs tendances. Elle nous dit les possibilités de leur réalisation. Ces faits peuvent être constatés par les sciences exactes et les mathématiques mais une fois qu'ils ont été réalisés par les hommes et non pas avant comme le pensent Weber et Aron.

\section{d. Le transfert des valeurs de l'éthique à l'entreprise}

L'assimilation de l'éthique par l'éthique de l'entreprise ne se trouve pas exempte de contradictions insurmontables : Soit qu'on analyse les conséquences d'une théorie éthique pour l'entreprise, soit qu'on observe l'aménagement d'une théorie éthique pour l'entreprise qui conduit les écrivains de l'éthique de l'entreprise à transformer le sens original de cette théorie éthique. 
Mais le transfert commet une autre erreur importante. Les écrivains de l'éthique de l'entreprise établissent une division fictive entre les valeurs morales de la société et les valeurs morales de l'entreprise ; ils font une sélection des valeurs de la société capitaliste en laissant les autres en deçà : par exemple l'égalité et la sécurité. D'autres valeurs comme la liberté, l'autonomie et la justice sont intégrées mais utilisées en accord avec les exigences de l'entreprise et avec le nouveau management participatif. Or, il semblerait qu'il s'agit de deux mondes qui fonctionnent avec une série de valeurs morales différentes : les valeurs de la société capitaliste et les valeurs de l'entreprise. Nous savons grâce á Orsoni de l'impossibilité d'avoir deux éthiques, une pour la société et l'autre pour l'entreprise (Leroux y Marciano, 1999: 197-205). Nous comprenons que le passage de la société à l'éthique de l'entreprise devrait prendre en compte les rapports de production, de pouvoir et d'autorité. Maintenant, en plus les écrivains de l'éthique de l'entreprise prétendent fonder une éthique de l'entreprise à travers une sélection des valeurs morales de la société capitaliste pour l'entreprise.

Même si les valeurs morales de la société capitaliste sont supposées par ces écrivains, le clivage qu'ils établissent demeure. Nous pensons que tout cet arrangement met en évidence le fait que le comportement moral dans la société et dans l'entreprise ne peut pas être le même. Il s'agit des rapports sociaux spécifiques. Autrement dit, cette division fictive reflète un fait réel. Dans l'entreprise on ne peut tenir les valeurs telles que la liberté, l'égalité, la justice, sans questionner le pouvoir, l'autorité et les processus mêmes de la production du profit. Ces valeurs doivent être substituées par les valeurs de responsabilité, de confiance, de courage, de tempérance, de respect, de loyauté, de créativité, de liberté, etc. Ce sont les valeurs que l'entreprise établit comme nécessaires pour son fonctionnement efficace. Rappelons que d'après Marx $(1965,1867)$ que la surface de la société est le véritable Eden des droits naturels de l'homme et du citoyen. Mais dès que les hommes franchissent le seuil de l'entreprise, leur comportement subit une transformation puisque l'égalité et la liberté sont substituées par l'autorité et la production de la plus-value (Marx 1965, 1867: 725-726). Il s'agit que du mode de vie capitaliste et de sa double morale qu'il comporte depuis sa naissance. 


\begin{tabular}{|c|c|}
\hline $\begin{array}{l}\text { LES V ALEURS DE LA } \\
\text { SOCIETE }\end{array}$ & $\begin{array}{c}\text { LES V ALEURS DE } \\
\text { L'ENTREPRISE }\end{array}$ \\
\hline $\begin{array}{l}\text { - Autonomie } \\
\text { - Bonheur } \\
\text { - } \text { Eiscipline } \\
\text { - Egalité } \\
\text { - Effort } \\
\text { - Fraternité } \\
\text { - Harmonie } \\
\text { - Humanité } \\
\text { - Indépendence } \\
\text { - Justice } \\
\text { - Optimisme } \\
\text { - Sécuritisme } \\
\text { - Tolérance } \\
\text { - Zèle }\end{array}$ & $\begin{array}{l}\text { - } \text { Autonomie } \\
\text { - } \text { Confiance } \\
\text { - } \text { Courage } \\
\text { - } \text { Dignité } \\
\text { - } \text { Fierté } \\
\text { - } \text { Foi } \\
\text { - Honnêteté } \\
\text { - } \text { Jumilité } \\
\text { - } \text { Liberté } \\
\text { - Loyauté } \\
\text { - } \text { Modestie } \\
\text { - } \text { Prudence } \\
\text { - Respect } \\
\text { - } \text { Responsabilité } \\
\text { - } \text { Transparité } \\
\text { - Tempérance } \\
\text { - Tolérance } \\
\text { - Innovation }\end{array}$ \\
\hline
\end{tabular}

Source: Les valeurs de la société capitaliste sont tirées du chapitres 3 et 4 de cette thèse du doctorat en sciences de gestion "L'éthique de l'entreprise capitaliste» (2003). Les valeurs de l'entreprise sont tirées des livres de Blanchard et Peale (1988); Le Tourneau (2000), Gélinier (1991), Even-Granboulan (1988), Daigne (1991), Claude (1998); Falise (1992) et Etchegoyen $(1990,1991)$ mais on les trouve citées partout par les écrivains de l'éthique de l'entreprise. Le tableau ne prétend pas être exhaustif et il doit être compris à partir des observations que nous avons fait.

\section{Resume et conclusion}

1. Dans cet article nous avons vu que l'éthique de l'entreprise use certaines parts des théories éthiques pour fonder recommandation sur ce que les affaires devraient être. Toutefois, les conséquences morales et les conséquences pratiques de cette application mènent à questionnements importants sur le pouvoir, l'autorité, la justice et l'égalité dans l'entreprise et sa responsabilité sociale.

2. Nous pensons que cela affecte le contenu orignal des théories éthiques mobilisées par les écrivains de l'éthique des affaires. Dans ce sens, le cas de M. Weber est 
notable. L'antinomie de l'éthique de la responsabilité et l'éthique de la conviction est détournée dans son sens original par les écrivains de l'éthique de l'entreprise. Ainsi, l'identification d'une part, de l'éthique de la responsabilité avec la morale de la raison et l'homme d'action, et d'autre part, de l'éthique de la conviction avec la morale du cour et l'homme sage ne correspondent pas à la pensée Weber; autant que l'identification de l'une ou de l'autre avec le bien et le mal; dieu et le diable. Et pourtant elles sont utilisées dans l'explication de l'agir du gestionnaire. Là, il faut dire que $\mathrm{M}$. Orsoni fait une interprétation plus proche à la pensée de Weber. Cette interprétation lui permet de proposer une dichotomie entre une morale instrumentale et une morale $d u$ cour plus près du comportement pratique du gestionnaire.

3. A partir de ce point de vue, le discours de l'éthique de l'entreprise effectue un transfert des valeurs morales de la société à l'entreprise dont les valeurs universelles restent comme présupposées. A leur place, l'éthique de l'entreprise reprend, ajoute et modifie les valeurs, celles qui sont nécessaires pour l'accomplissement du devoir dans l'entreprise. Il s'agit d'un arrangement des valeurs artificiel, mais qui met en évidence le fait réel de l'impossibilité de soutenir ces valeurs de la société capitaliste dans l'entreprise et sa gestion.

\section{Bibliographie}

Blanchard, K et Peale N.V ; Ethique et management. Réussir en restant intègre, Paris, Traduction par Marnat S ; Les Editions d'Organisation, 1988

Bergson, H ; Les deux sources de la morale et de la religion, Paris, PUF, 1932

Bergson, H ; L'évolution créatrice, Paris, PUF, 1998

Claude, J-F ; L'éthique au service du management, Paris. Les Éditions LIAISONS, 1998

Daigne, J-F ; L'éthique financière, Paris, PUF collection que sais-je ? numéro 2604, 1991 
Destutt, Comte de Tracy ; Eléments d'Idéologie, Première partie, L'Idéologie proprement dite, Troisième édition. Librairie Philosophique. J. VRIN, 1970 (1817)

De Feuilhade de Chauvin Tanneguy; Ethique et pouvoir dans l'entreprise, Paris, ESF éditeur, 1991

Dermagne, J ; Révolution chez les patrons? L'entreprise citoyenne, Paris, Bayard Editions, 1996

Etchegoyen, A ; La valse des Ethiques, Paris, Editions François Boudin, 1991

Even-Granboulan, G; Ethique et Economie. Quelle morale pour l'entreprise et le monde des affaires? Paris éditeur L'Harmattan, 1988

Farcy, H (présentateur) ; Vers une morale des affaires? L'Ethique du Responsable commercial, Paris, Institut catholique de Paris. Action populaire. Bibliothèque de la Recherche Sociale. SPES, 1969 Cas concrets introduit par Farcy, H

Ferry, J-M ; Habermas l'éthique de la communication, Paris, PUF, 1987

Frederick, R. E; La ética de los negocios. Aplicación a problemas específicos, México, Oxford University Press, Traducción del inglés Carril Villarreal M. del Pilar, 2001 (1999)

Floris, B ; La communication managériale. La modernisation symbolique des entreprises, Grenoble, PUG, 1996

Gélinier, O ; L'éthique des affaires. Halte à la dérive, Paris, Editions du Seuil, 1991

Habermas, $\mathrm{J}$; Morale et communication, Paris, Flammarion, Traduction et Introduction, par Bouchindhomme, C ; 1986 (1983)

Habermas, J ; De l'éthique de la discussion, Paris, Flammarion, Traduction et Introduction de l'allemand par Hunyadi, M ; 1992 (1991) 
Kant, E ; Métaphysique des mours, Paris, GF, Flammarion,1994, (1785)

Kosik, K ; La dialectique du concret, Traduit de l'allemand par Dangeville, R ; Paris, éditeur Maspero, 1970

Landier, H ; Vers l'entreprise intelligente. Dynamique du changement et mutation du changement, Paris, Editions Calmann-Lévy, 1991

Le Tourneau, P ; l'Ethique des affaires et du management au XXI siècle, Essai, Paris, éditeur Dalloz, 2000

Leroux, A et Marciano, A (sous la direction) ; Traité de philosophie économique, Paris, Economique, 1999

Marcuse, $\mathrm{H}$; L'homme unidimensionnel. Essai sur l'idéologie de la société industrielle avancée, Traduit de l'anglais par Wittig, M et l'auteur, 1968 Editions Minuit, 1968 (1964)

Marx, $\mathrm{K}$; Introduction générale à la critique de l'économie politique, Paris, Bibliothèque de la Pléiade. Euvres I Economie, édition établie, présentée et annotée par Maximilien Rubel. NRF Gallimard, 1965 (1857)

Marx, K ; Le Capital, livres I, II et III, Paris, Bibliothèque de la Pléiade. Euvres I Economie, édition établie, présentée et annotée par Maximilien Rubel. NRF Gallimard, 1965 (1867), 1968 (1885) (1894)

Marx, K et Engels, F ; L'Idéologie allemande, Paris, Bibliothèque de la Pléiade. Euvres III Philosophie, édition établie, présentée et annotée par Maximilien Rubel. NRF Gallimard, 1982 (1846)

Mercier, S ; L'éthique dans les entreprises, Paris, La Découverte, Collection Repères, 1999

Moussé, J ; Éthique et entreprises, Paris, éditeur Vuibert, 1993

Moussé, J ; Fondements d'une éthique professionnelle, Paris, Les Editons D’Organisation, 1989 
Orsoni, Jacques ; L'enseignant de gestion face à la morale d'entreprise, Revue française de gestion, $\mathrm{n}^{\circ}$ 74, juin, 1989, p. 114

Rochefoucauld (la) et Pietri, R ; L'entreprise et la société au service de l'homme, Paris. Editeur TEC \& DOC- Lavoisier. Institut de la Boétie, 1993

Solomon, R.C et Hanson, K.R ; La morale en affaires clé de la réussite, Paris, Éd. Organisation, 1989

Weber, M ; Economie et société, Paris, Plon 1995 (1921), T. 1 et 2

Weber, M ; Le savant et le politique, Paris, Plon, 10/18 traduit de l'allemand par Freud, J ; préface Aron, R ; 2001 (1919)

Wunenburger, J-J ; Questions d'éthique, Paris, PUF, Collection Premier Cycle, 1993 